Konrad-Zuse-Zentrum

für Informationstechnik Berlin

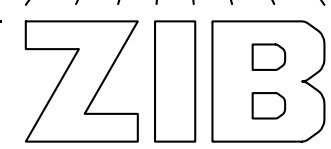

\title{
The Normal Graph Conjecture is true for Circulants
}




\title{
The Normal Graph Conjecture is true for Circulants
}

\author{
Annegret K. Wagler
}

January 3, 2005

\begin{abstract}
Normal graphs are defined in terms of cross-intersecting set families: a graph is normal if it admits a clique cover $\mathcal{Q}$ and a stable set cover $\mathcal{S}$ s.t. every clique in $\mathcal{Q}$ intersects every stable set in $\mathcal{S}$.

Normal graphs can be considered as closure of perfect graphs by means of co-normal products (Körner [6]) and graph entropy (Cziszár et al. [5]). Perfect graphs have been recently characterized as those graphs without odd holes and odd antiholes as induced subgraphs (Strong Perfect Graph Theorem, Chudnovsky et al. [3]). Körner and de Simone [9] observed that $C_{5}, C_{7}$, and $\bar{C}_{7}$ are minimal not normal and conjectured, as generalization of the Strong Perfect Graph Theorem, that every $C_{5}, C_{7}, \bar{C}_{7}$-free graph is normal (Normal Graph Conjecture, Körner and de Simone [9]).

We prove this conjecture for a first class of graphs that generalize both odd holes and odd antiholes, the circulants, by characterizing all the normal circulants.
\end{abstract}

\section{Introduction}

Normal graphs come up in a natural way in an information theoretic context $[7,5]$. A graph $G$ is called normal if it has the property of so-called "qualitative independence": $G$ admits a clique cover $\mathcal{Q}$ and a stable set cover $\mathcal{S}$ s.t. every clique in $\mathcal{Q}$ intersects every stable set in $\mathcal{S}$. (A set is a clique (resp. stable set) if its nodes are mutually adjacent (resp. non-adjacent).)

The interest in normal graphs is caused by the fact that they form, in many ways, a closure of the well-known class of perfect graphs. Berge introduced the latter class in 1960, motivated from Shannon's informationtheoretic problem of finding the zero-error capacity of a discrete memoryless 
channel [11]. Shannon's problem has a graph-theoretic formulation, regarding the asymptotic growth of the maximum cliques in the co-normal product $G^{n}$ of $G=(V, E)$, where $G^{2}$ has $V \times V$ as node set and

$$
\left\{\left(a_{1}, b_{1}\right),\left(a_{2}, b_{2}\right):\left(a_{1}, a_{2}\right) \in E \text { or }\left(b_{1}, b_{2}\right) \in E\right\}
$$

as edge set. The Shannon capacity of $G$ is

$$
C(G)=\lim _{n \rightarrow \infty} \frac{1}{n} \log \omega\left(G^{n}\right)
$$

where $\omega\left(G^{n}\right)$ denotes the size of a maximum clique in $G^{n}$. Shannon observed that $\omega\left(G^{n}\right)=(\omega(G))^{n}$ holds for graphs $G$ with $\omega(G)=\chi(G)$ which makes the otherwise difficult problem of determining $C(G)$ tractable $(\chi(G)$ denotes the least number of stable sets covering $V)$. Shannon observed further that the $C_{5}$ is the smallest graph $G$ with $\omega(G)<\chi(G)$ and that computing $C\left(C_{5}\right)$ is a difficult task, as for all chordless odd cycles $C_{2 k+1}$ with $k \geq 2$, called odd holes, see Figure 1.
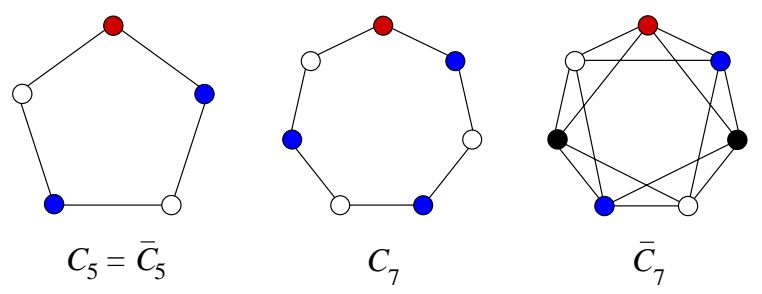

Figure 1: Small odd holes and odd antiholes

This led Berge [2] introduce perfect graphs as those graphs $G$, where $\omega\left(G^{\prime}\right)$ equals $\chi\left(G^{\prime}\right)$ for each induced subgraph $G^{\prime} \subseteq G$. Berge observed that, besides odd holes, also their complements, the odd antiholes $\bar{C}_{2 k+1}$ with $k \geq 2$, admit the property $\omega<\chi$, see Figure 1. (The complement $\bar{G}$ has the same node set as $G$ but two nodes are adjacent in $\bar{G}$ iff they are nonadjacent in $G$.) This motivated Berge's Strong Perfect Graph Conjecture:

$G$ is perfect $\Leftrightarrow G$ is odd hole- and odd antihole-free.

In particular, Berge claimed that the class of perfect graphs is closed under taking complements; this weaker conjecture has been proved in 1972 by Lovász [10]. The Strong Perfect Graph Conjecture stimulated the study of perfect graphs. It turned out that they have many fascinating properties and interesting relationships to other fields of scientific enquiry, see e.g. [1]. In 
a sequence of remarkable results based on the work of many graph-theorists Chudnovsky et al. [3] finally verified the conjecture; thus the Strong Perfect Graph Conjecture turned into the Strong Perfect Graph Theorem.

Normal graphs are closed under taking complements by definition, thus they share this property with perfect graphs. In this context it is natural to ask whether perfect and normal graphs are also closed under taking conormal products. Körner and Longo [7] showed that all co-normal products of $G$ are perfect if and only if $G$ is the union of disjoint cliques. However, all co-normal products of perfect and normal graphs are normal [6]; hence the class of normal graphs is the closure of perfect graphs w.r.t. taking co-normal products.

Another information-theoretic link between perfect and normal graphs has been established by means of graph entropy, see [12] for a survey. The entropy of a graph $G$ w.r.t. a probability distribution $p$ on its node set is

$$
H(G, p)=\limsup _{k \rightarrow \infty} \min \left\{\frac{1}{k} \log _{2} \chi\left(G^{k}[U]\right): U \subseteq V\left(G^{k}\right), \sum_{x \in U} p^{k}(x)>1-\varepsilon\right\} .
$$

The most important property of the graph-entropy is its sub-additivity w.r.t. graphs on the same node set, in particular for complementary graphs:

$$
H(p) \leq H(G, p)+H(\bar{G}, p) \forall p
$$

where $H(p)$ stands for the entropy of the complete graph, i.e., for the entropy of $p$ itself. Körner raised the question for which graphs $G$ the minimum $H(p)$ is attained, that means when equality holds rather than just sub-additivity. Körner and Longo [7] and Körner and Marton [8] proved

$$
H(p)=H(G, p)+H(\bar{G}, p) \text { for at least one } p>0 \Leftrightarrow G \text { is normal }
$$

and Cziszár et al. [5] obtained

$$
H(p)=H(G, p)+H(\bar{G}, p) \text { for all } p \Leftrightarrow G \text { is perfect }
$$

after a sequence of deep results on entropies of so-called convex corners. Hence normal graphs form also a superclass of perfect graphs by means of splitting graph entropies.

Since normal graphs are "weaker" perfect graphs in several ways, Körner and de Simone [9] asked finally for a similarity of the two classes in terms of forbidden subgraphs. Körner [6] showed that an odd hole $C_{2 k+1}$ is normal iff $k \geq 4$. In particular, $C_{5}$ and $C_{7}$ are not normal, and so neither $\bar{C}_{7}$ is. 
These three graphs are even minimally not normal since all of their proper induced subgraphs are perfect and, hence, normal. This led Körner and de Simone conjecture:

Conjecture 1 (Normal Graph Conjecture [9]) Graphs without any $C_{5}$, $C_{7}$, or $\bar{C}_{7}$ as induced subgraph are normal.

The validity of this conjecture would imply that the only minimally not normal graphs are precisely $C_{5}, C_{7}$, and $\bar{C}_{7}$ - as the only minimally imperfect graphs are precisely all odd holes and odd antiholes due to the Strong Perfect Graph Theorem.

Remark 2 In contrast to the Strong Perfect Graph Theorem, the validity of the Normal Graph Conjecture would provide us a sufficient condition for normality only, but no characterization: The non-existence of $C_{5}, C_{7}$, and $\bar{C}_{7}$ in a graph is not necessary for its normality. For example, the graphs $G_{1}$ and $G_{2}$ in Figure 2 are both normal (with the bold edges as clique covers and $\{\{1,3,5\},\{1,4,6\},\{2,4,5,7\}\}$ resp. $\{\{0,2,4,6\},\{0,3,5,7\},\{1,3,6\}\}$ as stable set covers) and so their complements are; but we have $C_{5} \subset G_{1}$, $C_{7} \subset G_{2}$, and $\bar{C}_{7} \subset \bar{G}_{2}$.

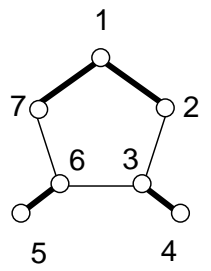

$G_{1}$

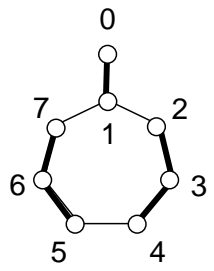

$G_{2}$

Figure 2: Normal graphs containing $C_{5}$ and $C_{7}$

Körner and de Simone [9] found a sufficient and necessary condition for the normality of connected triangle-free graphs: the existence of nice edge covers. (An edge cover $\mathcal{F}$ of a graph $G$ is nice if it is minimal w.r.t. set inclusion and if every odd cycle $C$ of $G$ has at least three nodes which are incident to an even number of edges in $E(C) \cap \mathcal{F}$; the bold edges in Figure 2 form nice edge covers.) However, this result in [9] does not prove the Normal Graph Conjecture for triangle-free graphs. In this paper we verify the conjecture for a first graph class: the circulants which are a common generalization of odd holes and odd antiholes. 
The paper is organized as follows: We introduce circulants in Section 2 and reformulate the Normal Graph Conjecture in an appropriate way in order to know for which circulants normality has to be shown. The main result is a characterization of all the normal circulants in Section 3. As a consequence we obtain that the Normal Graph Conjecture is true for circulant graphs. We conclude with some remarks and open problems.

\section{The Normal Graph Conjecture for Circulants}

We consider graphs with circular symmetry of their maximum cliques and stable sets, introduced by [4] as generalization of odd holes and odd antiholes. A circulant $C_{n}^{k}$ is a graph with nodes $1, \ldots, n$ where $i j$ is an edge if $i$ and $j$ differ by at most $k(\bmod n)$ and $i \neq j$.

We assume $k \geq 1$ and $n \geq 2(k+1)$ in the sequel in order to exclude the degenerated cases when $C_{n}^{k}$ is a stable set or a clique. $C_{n}^{1}$ is a hole and $C_{2 k+1}^{k-1}$ an odd antihole for $k \geq 2$. The circulants $C_{9}^{k}$ on nine nodes are depicted in Figure 3. The size of a maximum clique of the circulant $C_{n}^{k}$ is $k+1$ and the size of a maximum stable set, called stability number $\alpha$, is $\left\lfloor\frac{n}{k+1}\right\rfloor$. Unless stated otherwise, arithmetics are always performed modulo the number of nodes of the circulant involved in the computation.

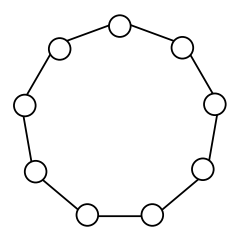

$C_{9}^{1}$

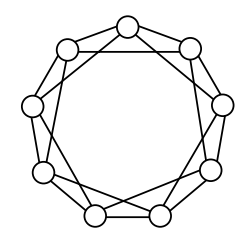

$C_{9}^{2}$

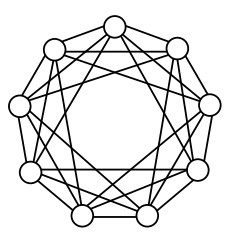

$C_{9}^{3}$

Figure 3: The circulants $C_{9}^{k}$

In order to prove the Normal Graph Conjecture for circulants, a first step is to figure out which circulants contain $C_{5}, C_{7}$, and $\bar{C}_{7}$ as induced subgraphs at all. This can be done with the help of the following result.

Theorem 3 (Trotter [13]) $C_{n^{\prime}}^{k^{\prime}}$ is an induced subgraph of $C_{n}^{k}$ if and only if $\frac{k+1}{k^{\prime}+1} n^{\prime} \leq n \leq \frac{k}{k^{\prime}} n^{\prime}$ holds.

Remark $4 C_{n^{\prime}}^{k^{\prime}} \subset C_{n}^{k}$ implies in particular $k^{\prime}<k$ and $n^{\prime}<n$ by [13]. 
Recalling $C_{5}=C_{5}^{1}, C_{7}=C_{7}^{1}$, and $\bar{C}_{7}=C_{7}^{2}$, we immediately obtain from Theorem 3 , for each fixed value of $k$, those numbers $n$ of nodes s.t. $C_{n}^{k}$ contains one of the subgraphs in question:

\section{Lemma 5}

(i) $C_{5}^{1} \subseteq C_{n}^{k}$ if and only if $\frac{5(k+1)}{2} \leq n \leq 5 k$.

(ii) $C_{7}^{1} \subseteq C_{n}^{k}$ if and only if $\frac{7(k+1)}{2} \leq n \leq 7 k$.

(iii) $C_{7}^{2} \subseteq C_{n}^{k}$ if and only if $\frac{7(k+1)}{3} \leq n \leq \frac{7 k}{2}$.

To illustrate the assertion of Lemma 5 look at the chart depicted in Figure 4. Every circle stands for a non-complete circulant $C_{n}^{k}$ with the respective values of $n$ and $k$. The dotted (resp. solid, resp. dashed) line indicates the range of circulants containing a $\bar{C}_{7}$ (resp. $C_{5}$, resp. $C_{7}$ ) as induced subgraph.

The overlap of the three ranges implies that, for each $k \geq 2$, the circulant $C_{n}^{k}$ is $\left(C_{5}, C_{7}, \bar{C}_{7}\right.$ )-free whenever $n<\frac{7(k+1)}{3}$ (lower bound for $C_{7}^{2} \subseteq C_{n}^{k}$ ) or $n>7 k$ (upper bound for $C_{7}^{1} \subseteq C_{n}^{k}$ ), see the grey-shaded range in Figure 4 . Since it is known from Körner [6] that all holes $C_{n}^{1}$ are normal if $n \neq 5,7$ we obtain as reformulation of the Normal Graph Conjecture for circulants:

\section{Conjecture 6 (Normal Graph Conjecture for Circulants)}

All circulants $C_{n}^{k}$ with $k \geq 2$ are normal for $n<\frac{7(k+1)}{3}$ or $n>7 k$.

Note that the circulants with $n<\frac{7(k+1)}{3}$ have stability number 2 by

$$
\alpha\left(C_{n}^{k}\right)=\left\lfloor\frac{n}{k+1}\right\rfloor<\left\lfloor\frac{\frac{7(k+1)}{3}}{k+1}\right\rfloor=\left\lfloor\frac{7(k+1)}{3(k+1)}\right\rfloor=2 .
$$

Hence we start in Subsection 3.1 with the case of circulants with $\alpha\left(C_{n}^{k}\right)=2$ and generalize these ideas in Subsection 3.2 further for the general case. This provides us a characterization of all normal circulants which shows particularly that the non-normal circulants are not $\left(C_{5}, C_{7}, \bar{C}_{7}\right)$-free - and finally verifies the Normal Graph Conjecture for circulants.

\section{The normal circulants}

For the sequel of this section, we consider circulants $C_{n}^{k}$ with $k \geq 2$ only (since the case of holes $C_{n}^{1}$ is trivial). In order to construct clique covers for normal graphs, we obviously need cliques maximal w.r.t set inclusion only. The following holds for maximal cliques of circulants: 


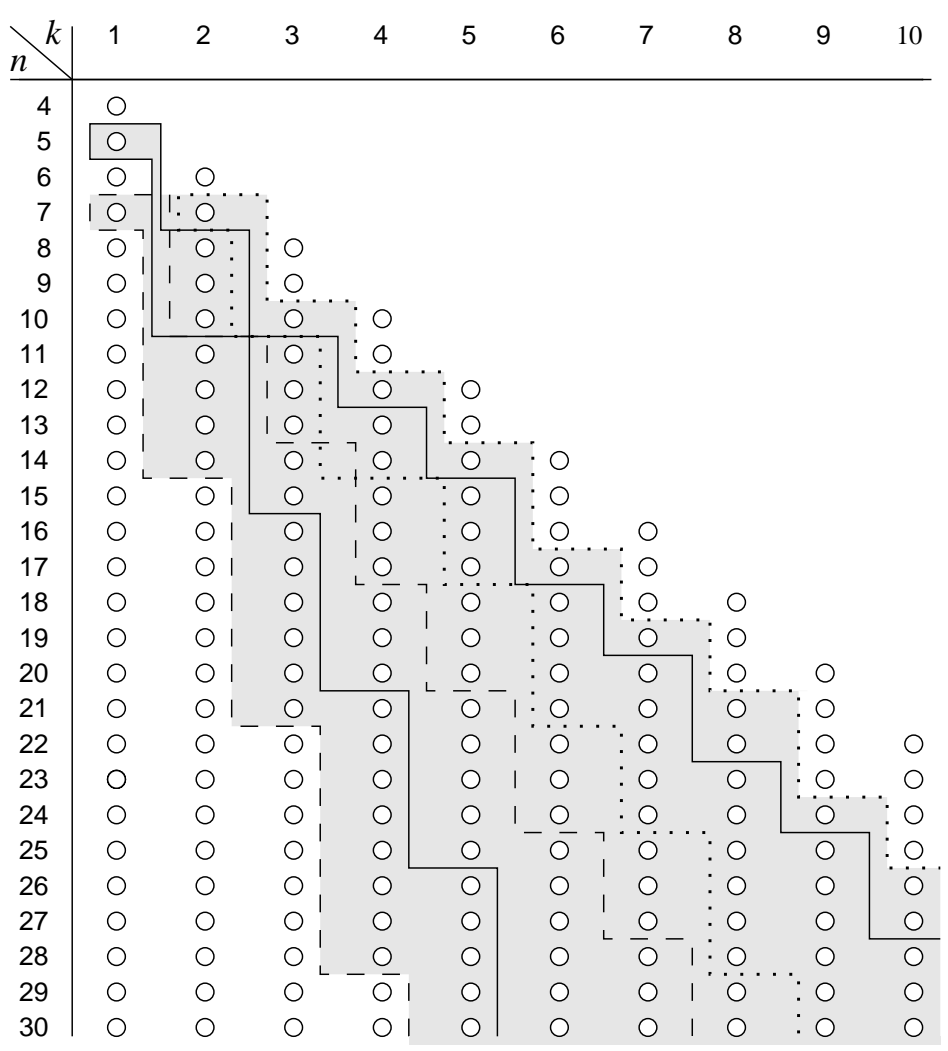

Figure 4: Circulants containing $C_{5}, C_{7}$, or $\bar{C}_{7}$

Lemma $7 A$ circulant $C_{n}^{k}$ with $k \geq 2$ has maximal cliques $Q$ with $|Q| \leq k$ only if $n \leq 3 k$.

Proof. Consider a maximal clique $Q \subseteq C_{n}^{k}$ with $|Q| \leq k$. Then $Q$ can neither be a single node nor a single edge, hence $Q$ must contain a triangle $x, y, z$. Furthermore, $Q$ is not contained in any maximum clique of $C_{n}^{k}$ (by the maximality of $Q$ ), hence $Q$ is not contained in any interval of $k+1=$ $\omega\left(C_{n}^{k}\right)$ consecutive nodes of $C_{n}^{k}$ in particular. The neighborhood $N(i)$ of every node $i$ of $C_{n}^{k}$ splits into two cliques $N^{-}(i)=\{i-k, \ldots, i-1\}$ and $N^{+}(i)=\{i+1, \ldots, i+k\}$ where all indices are taken modulo $n$. This implies $y=x+d \in N^{+}(x)$ and $z=x-d^{\prime} \in N^{-}(x)$ where $d+d^{\prime}+1>k+1$ holds, see Figure 5 .

Thus $y, z \in Q$ implies $z \in N^{+}(y)$ and we conclude: $y \leq x+k, z \leq$ $y+k \leq x+2 k$, and $x \leq z+k \leq x+3 k$ (where all indices are taken modulo 


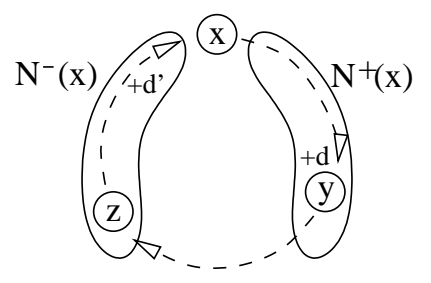

Figure 5: The neighborhood of $x$

$n$ again). Consequently, $n \leq 3 k$ is a necessary condition for $x, y, z$ to form a triangle. This implies that maximal but non-maximum cliques can appear in circulants $C_{n}^{k}$ with $n \leq 3 k$ only.

\section{$3.1 \quad$ The case $\alpha\left(C_{n}^{k}\right)=2$}

In this subsection, we characterize which circulants with stability number 2 are normal.

Lemma 8 The circulants $C_{n}^{k}$ with $n \leq 3 k$ are normal.

Proof. We show normality in this case by explicitely constructing a clique cover $\mathcal{Q}$ and a cross-intersecting stable set cover $\mathcal{S}$.

Choose a family of three pairwise intersecting maximum cliques, e.g. $\mathcal{Q}=$ $\left\{Q^{+}(1), Q^{+}(k+1), Q^{+}(2 k+1)\right\}$ where $Q^{+}(i)=\{i, \ldots, i+k\}$ and all indices are taken modulo $n$, see Figure 6 (recall $n \geq 2(k+1)$ ). By construction, we have

$$
\begin{aligned}
Q^{+}(1) \cap Q^{+}(k+1) & =\{k+1\}, \\
Q^{+}(k+1) \cap Q^{+}(2 k+1) & =\{2 k+1\}, \\
Q^{+}(2 k+1) \cap Q^{+}(1) & =\{1, \ldots, 3 k+1 \bmod n\}
\end{aligned}
$$

(thus the intersection contains in the latter case at least node 1 by $n \leq 3 k$ ), see Figure 6. In particular, $\mathcal{Q}$ is a clique cover of $C_{n}^{k}$.

We construct a cross-intersecting stable set cover $\mathcal{S}$ by choosing as stable sets the nodes $x$ belonging to one clique $Q \in \mathcal{Q}$ only together with the minimal non-neighbor of $x$ in the intersection of the other two cliques in $\mathcal{Q}-Q$. Thus, we construct stable sets

$$
S_{x}:= \begin{cases}\{x, 2 k+1\} & \text { if } x \in\{3 k+2 \bmod n, \ldots, k\} \\ \{x, y\} & \text { if } x \in\{k+2, \ldots, 2 k\} \\ \{x, k+1\} & \text { if } x \in\{2 k+2, \ldots, n\}\end{cases}
$$




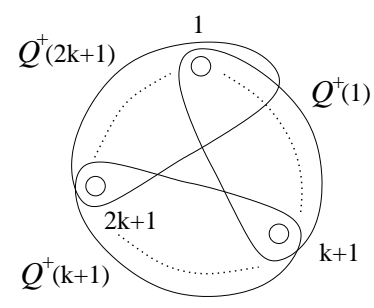

Figure 6: The clique cover of $C_{n}^{k}$ with $n \leq 3 k$

with $y \in\{1, \ldots, 3 k+1 \bmod n\}$ minimal non-neighbor of $x$ in $Q^{+}(2 k+1) \cap$ $Q^{+}(1)$ (i.e., $y=1$ if $x \leq n-k$ up to $y=3 k+1 \bmod n$ if $x=2 k$ ), see Figure 6 again. By construction, every set $S_{x}$ is stable and meets all three cliques in $\mathcal{Q}$. The union $\mathcal{S}$ of these sets $S_{x}$ covers all nodes of $C_{n}^{k}$ (this is obvious for the nodes $3 k+2 \bmod n, \ldots, n$ and follows for $1, \ldots, 3 k+1 \bmod n$ by choosing the minimal non-neighbor of $x$ in $\left.Q^{+}(2 k+1) \cap Q^{+}(1)\right)$. Hence $\mathcal{Q}$ and $\mathcal{S}$ are the set families showing normality of $C_{n}^{k}$ with $n \leq 3 k$.

Example 9 The set families $\mathcal{Q}$ and $\mathcal{S}$ for $C_{11}^{4}$ constructed as above are the following: $\mathcal{S}=\{\{3,9\},\{4,9\},\{6,1\},\{7,1\},\{8,2\},\{10,5\},\{11,5\}\}$ and $\mathcal{Q}=\left\{Q^{+}(1), Q^{+}(5), Q^{+}(9)\right\}$ (see Figure 7 ).

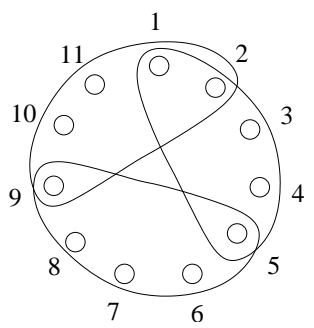

Figure 7: The clique cover of $C_{11}^{4}$

Due to $\frac{7(k+1)}{3} \leq 3 k+1$, Lemma 8 proofs already one part of the Normal Graph Conjecture for circulants. The above construction, however, cannot be extended to the remaining cases of circulants with stability number 2 :

Lemma 10 For all $k \geq 2$, the circulants $C_{3 k+1}^{k}$ and $C_{3 k+2}^{k}$ are not normal.

Proof. Assume in contrary $C_{n}^{k}$ with $n \in\{3 k+1,3 k+2\}$ has a clique cover $\mathcal{Q}$ and a cross-intersecting stable set cover $\mathcal{S}$. Lemma 7 implies that 
all maximal cliques of $C_{n}^{k}$ have maximum size $k+1$ by $n>3 k$. Hence, $\mathcal{Q}$ consists of cliques $Q^{+}(i)=\{i, \ldots, i+k\}$ only.

In particular, there are two disjoint cliques $Q$ and $Q^{\prime}$ in $\mathcal{Q}$ : Otherwise, every two cliques of $\mathcal{Q}$ would intersect. For $|\mathcal{Q}|=2$, we would obtain $n \leq 2 k+1$, a contradiction to $k \geq 2$ and $n>3 k$. For $|\mathcal{Q}| \geq 3$, the cliques intersect pairwise only if $n \leq 3 k$, a contradiction to $n>3 k$ again.

The two disjoint cliques $Q$ and $Q^{\prime}$ cannot cover all nodes of $C_{n}^{k}$ with $k \geq 2$ by $n \geq 3 k+1>2(k+1)=\left|Q \cup Q^{\prime}\right|$. Hence, there is a node $x \notin Q \cup Q^{\prime}$. Due to $\alpha\left(C_{n}^{k}\right)=2$, there is no stable set in $\mathcal{S}$ which contains $x$ and meets both cliques $Q$ and $Q^{\prime}$ (such a stable set had to contain three nodes). Thus, $C_{n}^{k}$ with $n=3 k+1,3 k+2$ and $k \geq 2$ does not admit a clique cover and a cross-intersecting stable set cover and is, therefore, not normal.

Remark 11 The complements $\bar{C}_{n}^{k}$ of the circulants with stability number 2 are triangle-free. Hence the complements are normal if and only if they admit a nice edge cover due to Körner and de Simone [9]. Lemma 8 constructs, therefore, stable set covers corresponding to nice edge covers in $\bar{C}_{\leq 3 k}^{k}$ whereas Lemma 10 shows that $\bar{C}_{3 k+1}^{k}$ and $\bar{C}_{3 k+2}^{k}$ do not admit any nice edge cover. The proofs of the two lemmas are, however, easier than verifying directly whether nice edge covers exist in the complements.

\subsection{The general case}

We generalize the clique covers from the previous subsection to the case $n \geq 3 k+3$. Note that in this case all maximal cliques of $C_{n}^{k}$ have maximum size $k+1$ by Lemma 7 . For $n \geq 3 k+1$, there are no pairwise intersecting cliques covering all nodes, but cyclic intersecting cliques with that property.

We call a clique cover $\mathcal{Q}=\left\{Q_{1}, \ldots, Q_{l}\right\}$ of $C_{n}^{k}$ cyclic if each clique $Q_{i}$ has a non-empty intersection with precisely the cliques $Q_{i-1}$ and $Q_{i+1}$ (where the indices are taken modulo $l$ ).

A circulant $C_{n}^{k}$ has in general several cyclic clique covers $\mathcal{Q}=\left\{Q_{1}, \ldots, Q_{l}\right\}$ of the same size consisting of maximum cliques, see Figure 8, namely as many as $C_{n}^{k}$ has different holes $C_{l}^{1}$ as induced subgraphs: Denote by $q_{i}$ the first node in $Q_{i}$, then $q_{i}$ is adjacent to $q_{i-1}$ and $q_{i+1}$ but not to $q_{j}$ with $i+1<j<i-1 \bmod l$ by definition; thus $q_{1}, \ldots, q_{l}$ induce an $l$-hole in $C_{n}^{k}$. On the other hand, consider $C_{l}^{1} \subseteq C_{n}^{k}$ with nodes $q_{1}, \ldots, q_{l}$ and the maximum cliques $Q^{+}\left(q_{i}\right)=\left\{q_{i}, \ldots, q_{i}+k\right\}$ of $C_{n}^{k}$ starting in $q_{i}$. A result of Trotter [13] shows that $Q^{+}\left(q_{i}\right)$ contains precisely two nodes of $C_{l}^{1}$, namely $q_{i}$ and $q_{i+1}$; thus $\mathcal{Q}=\left\{Q^{+}\left(q_{1}\right), \ldots, Q^{+}\left(q_{l}\right)\right\}$ is a cyclic clique cover. Hence 

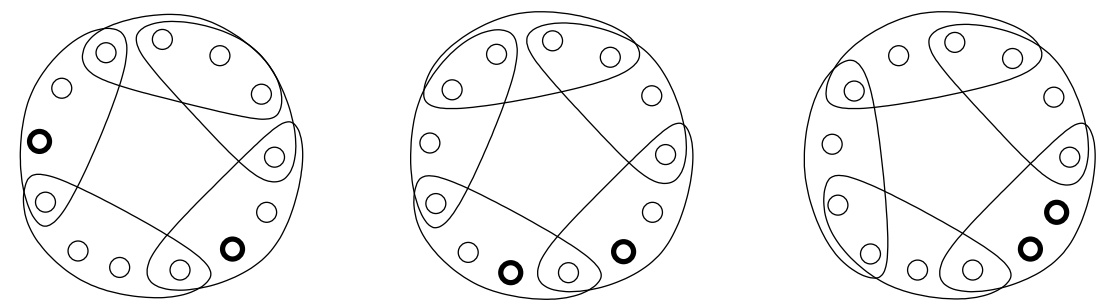

Figure 8: Different cyclic clique covers of size 5 in $C_{13}^{3}$

cyclic clique covers of size $l$ and holes $C_{l}^{1} \subseteq C_{n}^{k}$ correspond to each other and by Theorem 3 we have

$$
C_{l}^{1} \subseteq C_{n}^{k} \text { iff } \frac{(k+1)}{2} l \leq n \leq \frac{k}{1} l
$$

implying the following:

Lemma $12 C_{n}^{k}$ with $k \geq 2$ admits, for each $t \geq 2$, a cyclic clique cover $\mathcal{Q}$ of size $2 t-1$ iff

$$
\frac{(k+1)}{2}(2 t-1) \leq n \leq \frac{k}{1}(2 t-1) .
$$

Let $q(x, \mathcal{Q})$ stand for the number of cliques in $\mathcal{Q}$ containing node $x$. By the definition of $\mathcal{Q}$, we have $q(x, \mathcal{Q}) \in\{1,2\}$ for all nodes $x$ (since $\mathcal{Q}$ covers all nodes but no three cliques intersect). We call $x$ a 1-node (resp. 2-node) w.r.t. $\mathcal{Q}$ if $q(x, \mathcal{Q})=1$ (resp. $q(x, \mathcal{Q})=2$ ) holds.

Note that, for a cyclic clique cover $\mathcal{Q}$ containing $2 t-1$ cliques of size $k+1$ each, the lower bound $\frac{(k+1)}{2}(2 t-1) \leq n$ in Lemma 12 is attained if there are 2-nodes only whereas the upper bound $n \leq \frac{k}{1}(2 t-1)$ guarantees that there is at least one 2-node in the intersection of two consecutive cliques.

The next step is to construct cross-intersecting stable sets and to show that they cover all nodes. The idea goes as follows: $\mathcal{Q}$ contains $2 t-1$ cliques. If a 1-node $x$ belongs to $Q \in \mathcal{Q}$, then $\mathcal{Q}-Q$ consists of $2 t-2$ cliques or, in other words, of $t-1$ pairs of intersecting cliques. We denote by $S(x, \mathcal{Q})$ a $t$-set containing $x$ and one node from the intersection of the $t-1$ pairs of cliques, see Figure 9 . Thus $S(x, \mathcal{Q})$ intersects all cliques in $\mathcal{Q}$ by construction; we have to show that there exist stable sets $S(x, \mathcal{Q})$ whose union covers all nodes of $C_{n}^{k}$. 
Lemma 13 Consider a cyclic clique cover $\mathcal{Q}$ of $C_{n}^{k}$ of size $2 t-1$ where $t(k+1) \leq n \leq(2 t-1) k$ and $k, t \geq 2$. For each 1-node $x$ w.r.t. $\mathcal{Q}$ of $C_{n}^{k}$ there is a stable set $S(x, \mathcal{Q})$ of size $t$ in $C_{n}^{k}$.

Proof. $C_{n}^{k}$ admits by $\frac{(2 t-1)(k+1)}{2}<t(k+1)$ a cyclic clique cover $\mathcal{Q}=$ $\left\{Q_{1}, \ldots, Q_{2 t-1}\right\}$ due to Lemma 12. Furthermore, $t(k+1) \leq n$ guarantees that $C_{n}^{k}$ contains stable sets of size $t$ by $t \leq \alpha\left(C_{n}^{k}\right)=\left\lfloor\frac{n}{k+1}\right\rfloor$.

Consider a 1-node $x$ of $C_{n}^{k}$ and assume w.l.o.g. that $x$ belongs to $Q_{1} \in \mathcal{Q}$. We construct a stable set $S(x, \mathcal{Q})=\left\{x, x_{1}, \ldots, x_{t-1}\right\}$ s.t. $x_{i} \in Q_{2 i} \cap Q_{2 i+1}$ for $1 \leq i \leq t-1$, see Figure 9 .

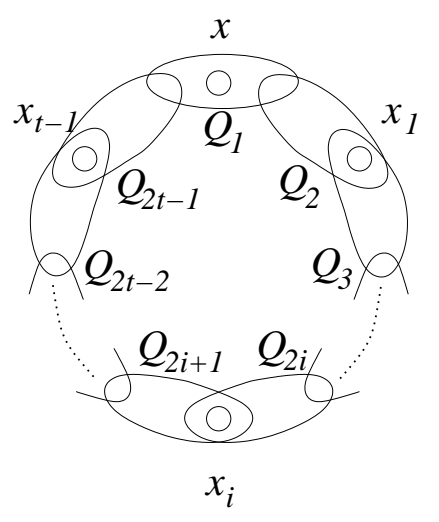

Figure 9: Constructing the stable set $S(x, \mathcal{Q})$ for $x \in Q_{1}$

Since $x \in Q_{1}-Q_{2}$, there is a non-neighbor of $x$ in $Q_{2} \cap Q_{3}$ (at least the last node in $Q_{2}$ is not adjacent to $x$ but belongs to $Q_{3}$ ). We choose $x_{1}=x+(k+1)+d_{1} \in Q_{2} \cap Q_{3}$ with $d_{1} \in \mathbb{N} \cup\{0\}$ minimal.

In order to construct $x_{i}$ from $x_{i-1}$ for $2 \leq i \leq t-1$, notice that we have $x_{i-1} \in Q_{2 i-2} \cap Q_{2 i-1}$, in particular $x_{i-1} \in Q_{2 i-1}-Q_{2 i}$. As before, there is a non-neighbor of $x_{i-1}$ in $Q_{2 i} \cap Q_{2 i+1}$ and we choose $x_{i}=x_{i-1}+(k+1)+d_{i} \in$ $Q_{2 i} \cap Q_{2 i+1}$ with $d_{i} \in \mathbb{N} \cup\{0\}$ minimal.

Then $S(x, \mathcal{Q})$ is a stable set if $x_{t-1}$ and $x$ are non-adjacent (all other nodes are non-adjacent by construction).

If $d_{i}=0$ for $1 \leq i \leq t-1$, then $x_{t-1}=x+(t-1)(k+1)$. Hence, there are at least $k+1$ nodes between $x_{t-1}$ and $x$ (in increasing order modulo $n$ ) due to $n \geq t(k+1)$ and we are done. Otherwise, let $j$ be the smallest index s.t. $d_{j}>0$. Then $x_{j}$ is the first node in $Q_{2 j+1}$ since we choose $d_{j}$ minimal: By $x_{j-1} \notin Q_{2 j}$, we have $x_{j-1}+(k+1) \in Q_{2 j}$. The only reason for choosing $d_{j}>0$ was, therefore, $x_{j-1}+(k+1)+d_{j}^{\prime} \notin Q_{2 j+1}$ for all $0 \leq d_{j}^{\prime}<d_{j}$ by the 
minimality of $d_{j}$. Hence, $x_{j}$ is indeed the first node in $Q_{2 j+1}$. This implies that its first non-neighbor is the node $x_{j}+(k+1)$ belonging to $Q_{2 j+2}-Q_{2 j+1}$ and $x_{j+1}=x_{j}+(k+1)+d_{j+1} \in Q_{2 j+2} \cap Q_{2 j+3}$ is, by the minimality of $d_{j+1}$, the first node of $Q_{2 j+3}$. The same argumentation shows that every further $x_{i}$ with $i>j+1$ is the first node in $Q_{2 i+1}$; in particular, $x_{t-1}$ is the first node of $Q_{2 t-1}$. Hence, $x \in Q_{1}-Q_{2 t-1}$ shows that $x_{t-1}$ and $x$ are non-adjacent. Thus $S(x, \mathcal{Q})=\left\{x, x_{1}, \ldots, x_{t-1}\right\}$ is a stable set of size $t$ and intersects all cliques of $\mathcal{Q}$ by $x \in Q_{1}$ and $x_{i} \in Q_{2 i} \cap Q_{2 i+1}$ for $1 \leq i \leq t-1$.

Remark 14 Lemma 13 implies that there is, for each 1-node $x$, at least one stable set $S(x, \mathcal{Q})$. In general, there may be several such sets: Consider, e.g., the cyclic clique covers $\mathcal{Q}$ of $C_{13}^{3}$ indicated in Figure 8. There are two stable sets $S(x, \mathcal{Q})$ for the bold 1-nodes $x$.

It is left to show that the union of all stable sets $S(x, \mathcal{Q})$ covers the circulant.

Lemma 15 Consider a cyclic clique cover $\mathcal{Q}$ of $C_{n}^{k}$ of size $2 t-1$ where $t(k+1) \leq n \leq(2 t-1) k$ and $k, t \geq 2$. Then the union $\mathcal{S}$ of the stable sets $S(x, \mathcal{Q})$, where $x$ is a 1-node of $C_{n}^{k}$ w.r.t $\mathcal{Q}$, covers all nodes of $C_{n}^{k}$.

Proof. Assume in contrary that there is a node $y$ in $C_{n}^{k}$ not covered by $\mathcal{S}$. Then there is no stable set $S(x, \mathcal{Q})$ with $y \in S(x, \mathcal{Q})$. In particular, $y$ is a 2-node w.r.t. $\mathcal{Q}$ by Lemma 13. W.l.o.g. let $y \in Q_{1} \cap Q_{2 t-1}$. We first show $y_{l}=y+l(k+1) \in Q_{2 l+1}$ for $0 \leq l \leq t-2$. Clearly, we have $y=y_{0}=y+$ $0(k+1) \in Q_{1}$ by assumption and prove that $y_{i-1}=y+(i-1)(k+1) \in Q_{2 i-1}$ implies $y_{i}=y+i(k+1) \in Q_{2 i+1}$ for $1 \leq i \leq t-2$.

If there is a 1-node $x$ in $Q_{2 i} \backslash\left(Q_{2 i-1} \cup Q_{2 i+1}\right)$, then $x$ is adjacent to $y_{i-1}=y+(i-1)(k+1)$, see Figure 10(a) (otherwise, there is a stable set $S(x, \mathcal{Q})$ containing $x$ and $y_{0}, \ldots, y_{i-1}$ in contradiction to our assumption) and $x<y+i(k+1)$ yields $y_{i}=y+i(k+1) \in Q_{2 i+1}$.

If $Q_{2 i} \backslash\left(Q_{2 i-1} \cup Q_{2 i+1}\right)=\emptyset$, then $y_{i}=y+i(k+1)$ clearly belongs to $Q_{2 i+1}$ (since we have $y_{i-1}=y+(i-1)(k+1) \in Q_{2 i-1}$ and $\left|Q_{2 i-1}\right|=k+1$ ).

In particular, we have $y_{t-2}=y+(t-2)(k+1) \in Q_{2 t-3}$. Any 1-node $x$ in $Q_{2 t-2} \backslash\left(Q_{2 t-3} \cup Q_{2 t-1}\right)$ is adjacent to $y_{t-2}$ or to $y$, see Figure 10(b) (otherwise, $x$ together with $y_{0}, \ldots, y_{t-2}$ would be a set $S(x, \mathcal{Q}) \in \mathcal{S}$ in contradiction to our assumption). We distinguish three cases:

If $x$ is adjacent to $y$, then $x>y-(k+1)=y_{-1}$ follows and $y_{-1}$ is adjacent to $y_{t-2}$ : either $y_{-1}$ belongs to $Q_{2 t-3}$ or is as 1-node adjacent to $y_{t-2}$; thus, $y_{-1}=y-(k+1) \leq y+(t-2)(k+1)+k=y_{t-2}+k$ implies $y \leq y+(t-1)(k+1)+k$. 


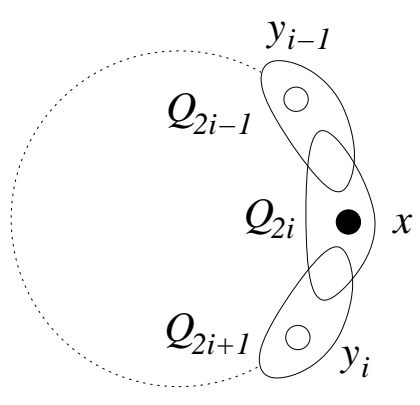

(a)

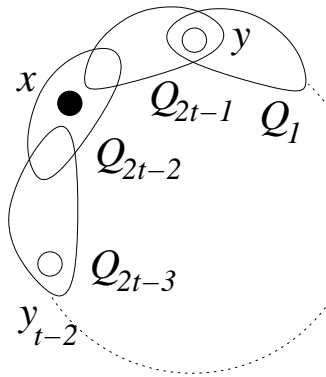

(b)

Figure 10: Constructing the nodes $y_{i} \in Q_{2 i+1}$

If $x$ is adjacent to $y_{t-2}$, we obtain $x<y_{t-2}+(k+1)=y_{t-1}$ and $y_{t-1}$ either belongs to $Q_{2 t-1}$ or is a 1-node adjacent to $y$; here, $y_{t-1} \geq y-k$ and, therefore, $y+(t-1)(k+1) \geq y-k$ holds.

The non-existence of a 1-node in $Q_{2 t-2}$ implies $y_{t-1} \in Q_{2 t-2}$ and, therefore, $y_{t-1} \geq y-k$ follows again.

All three cases imply $n \leq(t-1)(k+1)+k$. By $n \geq t(k+1)$, we obtain

$$
t(k+1) \leq(t-1)(k+1)+k
$$

yielding the final contradiction. Hence the union $\mathcal{S}$ of the stable sets $S(x, \mathcal{Q})$, where $x$ is a 1-node of $C_{n}^{k}$ w.r.t $\mathcal{Q}$, covers all nodes of $C_{n}^{k}$.

Since each $S(x, \mathcal{Q})$ meets all cliques in $\mathcal{Q}$ by construction, $\mathcal{S}$ is the required cross-intersecting stable set cover. Thus Lemma 12, Lemma 13, and Lemma 15 together imply:

Theorem 16 A circulant $C_{n}^{k}$ with $k \geq 2$ admits for each $t \geq 2$

- a cyclic clique cover $\mathcal{Q}$ of size $2 t-1$ and

- a cross-intersecting stable set cover $\mathcal{S}$ of stable $t$-sets if $t(k+1) \leq n \leq(2 t-1) k$ holds.

Remark 17 Theorem 16 includes the case $t=2$ from Lemma 8: every circulant $C_{n}^{k}$ with $n \leq 3 k$ has a cyclic clique cover of size 3 and a crossintersecting cover of stable 2 -sets. 
Theorem 16 shows that circulants $C_{n}^{k}$ are normal for certain numbers $n$ of nodes - depending on the values of $k$ and $t$. We finally have to check whether, for fixed $k$, there are gaps between the ranges $t(k+1) \leq n \leq(2 t-1) k$ and $(t+1)(k+1) \leq n \leq(2 t+1) k$ of normal circulants for different values of $t \geq 2$, see Figure 11. The dotted (resp. solid, resp. dashed) line indicates the range for $t=2$ (resp. $t=3$, resp. $t=4)$. There is no gap between the two ranges if

$$
\begin{aligned}
(t+1)(k+1) & \leq(2 t-1) k+1 \\
t k+t+k+1 & \leq 2 t k-k+1 \\
\frac{2 k}{k-1} & \leq t .
\end{aligned}
$$

This is true for $k=2$ if $t \geq 4$ and for $k \geq 3$ if $t \geq 3$, hence we obtain:

Lemma 18 The ranges $t(k+1) \leq n \leq(2 t-1) k$ and $(t+1)(k+1) \leq n \leq$ $(2 t+1) k$ overlap for $k=2$ if $t \geq 4$ and for $k \geq 3$ if $t \geq 3$.

Thus Theorem 16 and Lemma 18 show the normality for all circulants $C_{n}^{k}$ with $k \geq 2$ except the cases $n=3 k+1,3 k+2$ (gap between the ranges for $t=2$ and $t=3$ ) and $C_{11}^{2}$ (gap between the ranges for $t=3$ and $t=4$ ), see Figure 11. We proved in Lemma 10 that $C_{3 k+1}^{k}$ and $C_{3 k+2}^{k}$ are not normal for all $k \geq 2$; in order to characterize completely which circulants are normal and which are not, it is only left to check $C_{11}^{2}$.

Lemma 19 The circulant $C_{11}^{2}$ is not normal.

Proof. Assume in contrary $C_{11}^{2}$ admits a clique cover $\mathcal{Q}$ and a crossintersecting stable set cover $\mathcal{S}$. Lemma 7 implies that all maximal cliques of $C_{11}^{2}$ are of the form $Q^{+}(i)=\{i, i+1, i+2\}$.

First, $\mathcal{Q}$ must not contain three disjoint cliques $Q, Q^{\prime}, Q^{\prime \prime}$ : These three cliques cannot cover all nodes of $C_{11}^{2}$ by $11>9=\left|Q \cup Q^{\prime} \cup Q^{\prime \prime}\right|$. Hence, there is a node $x \notin Q \cup Q^{\prime} \cup Q^{\prime \prime}$ but due to $\alpha\left(C_{11}^{2}\right)=3$, there is no stable set in $\mathcal{S}$ containing $x$ and meeting all three cliques $Q, Q^{\prime}, Q^{\prime \prime}$.

Thus $\mathcal{Q}$ contains at most two disjoint cliques $Q$ and $Q^{\prime}$. They must not be consecutive (see Figure 12(a)): If $Q=\{1,2,3\}$ and $Q^{\prime}=\{4,5,6\}$ we need a clique $Q^{\prime \prime}$ among $\{7,8,9\},\{8,9,10\},\{9,10,11\}$ in order to cover node 9 , but $Q, Q^{\prime}, Q^{\prime \prime}$ would be disjoint in any case.

There is no possibility for avoiding two consecutive disjoint cliques: Suppose $Q=\{1,2,3\}$. If $Q^{\prime}=\{5,6,7\}$ (Figure 12(b)), then $\{8,9,10\},\{9,10,11\}$ $\notin \mathcal{Q}$ follows. But in order to cover the nodes 9 and 10, we need the two consecutive cliques $\{7,8,9\}$ and $\{10,11,1\}$. If $Q^{\prime}=\{6,7,8\}$ (Figure 12(c)), then $\{4,5,6\},\{3,4,5\} \notin \mathcal{Q}$ follows. Thus we need the two consecutive cliques 


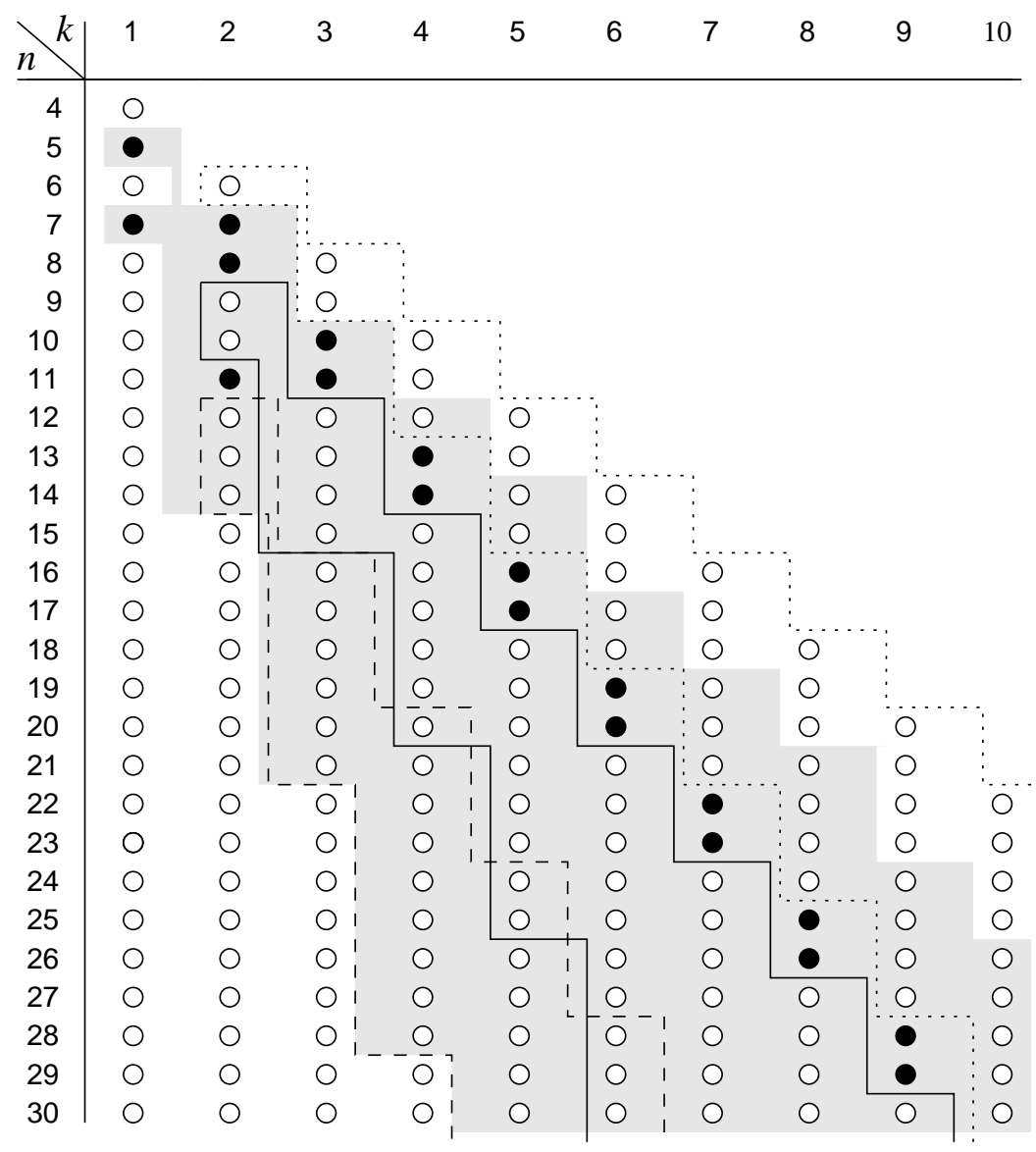

Figure 11: The ranges of normal circulants for $t=2,3,4$

$\{2,3,4\}$ and $\{5,6,7\}$ in order to cover the nodes 4 and 5 which yields the final contradiction.

As a consequence, we are able to characterize all the normal circulants:

Theorem 20 A circulant $C_{n}^{k}$ is normal if and only if

- $k=1$ and $n \neq 5,7$,

- $k=2$ and $n \neq 7,8,11$,

- $k \geq 3$ and $n \neq 3 k+1,3 k+2$. 


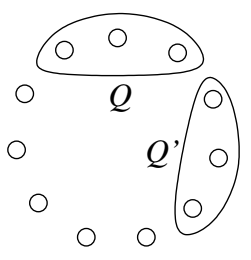

(a)

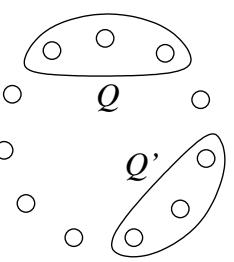

(b)

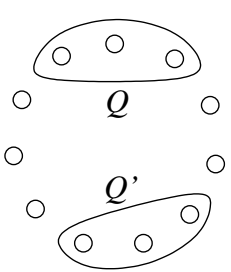

(c)

Figure 12: Two disjoint cliques $Q$ and $Q^{\prime}$ in $C_{11}^{2}$

Since all the non-normal circulants are not $\left(C_{5}, C_{7}, \bar{C}_{7}\right)$-free (see the black circles in Figure 11), Theorem 20 finally verifies the Normal Graph Conjecture for circulants; since normality is closed under taking complements, we obtain the same assertion for the complementary class:

Corollary 21 The Normal Graph Conjecture is true for circulants and their complements.

\section{Concluding Remarks}

In this paper we verified the Normal Graph Conjecture for a first graph class, the circulants, and for their complements. Next steps could be to treat the conjecture for partitionable graphs (as a different generalization of odd holes and odd antiholes) and for circular-arc graphs (as generalization of circulants).

However, even proving the Normal Graph Conjecture in general would not yield a characterization of normal graphs, see Remark 2, since induced subgraphs of normal graphs are not necessarily normal. In order to get a better analogy with perfect graphs, Körner and de Simone [9] introduced a hereditary property by defining strongly normal graphs as those normal graphs whose induced subgraphs are all normal. In terms of strongly normal graphs the Normal Graph Conjecture is equivalent to the following:

Conjecture 22 (Strongly Normal Graph Conjecture [9]) A graph G is strongly normal if and only if neither $G$ nor its complement $\bar{G}$ contain any $C_{5}$ or $C_{7}$ as induced subgraph.

The interest of this conjecture lies in the fact that it would immediately lead to a polynomial time recognition algorithm for strongly normal graphs. 


\section{References}

[1] J. Ramirez-Alfonsin and B. Reed, Perfect Graphs, Wiley (2001)

[2] C. Berge, Färbungen von Graphen, deren sämtliche bzw. deren ungerade Kreise starr sind, Wiss. Zeitschrift der Martin-Luther-Universität Halle-Wittenberg 10 (1961) 114-115.

[3] M. Chudnovsky, N. Robertson, P. Seymour, and R. Thomas, The Strong Perfect Graph Theorem, http://www.math.gatech.edu/ ${ }^{\sim}$ thomas/spgc.ps.gz

[4] V. Chvátal, On the Strong Perfect Graph Conjecture, J. Combin. Theory B 20 (1976) 139-141.

[5] I. Cziszár, J. Körner, L. Lovász, K. Marton, and G. Simonyi. Entropy splitting for antiblocking corners and perfect graphs, Combinatorica 10 (1990) 27-40.

[6] J. Körner, An Extension of the Class of Perfect Graphs, Studia Math. Hung. 8 (1973) 405-409.

[7] J. Körner and G. Longo, Two-step encoding of finite memoryless sources, IEEE Trans. Inform. Theory 19 (1973) 778-782.

[8] J. Körner and K. Marton, Graphs that split entropies, SIAM J. Discrete Math. 1 (1988) 71-79.

[9] J. Körner and C. de Simone, On the Odd Cycles of Normal Graphs, Discrete Appl. Math. 94 (1999) 161-169.

[10] L. Lovász, Normal hypergraphs and the weak perfect graph conjecture, Discrete Math. 2 (1972) 253-267.

[11] C.E. Shannon. The zero-error capacity of a noisy channel, IRE Trans. Inform. Theory 2 (1956) 8-19.

[12] G. Simonyi. Perfect Graphs and Graph Entropy, In: Perfect Graphs, J.L. Ramirez-Alfonsin und B.A. Reed (eds.), Wiley, pages 293-328, 2001.

[13] L.E. Trotter, jr., A Class of Facet Producing Graphs for Vertex Packing Polyhedra. Discrete Math. 12 (1975) 373-388 